\title{
Low-dose gadobenate dimeglumine-enhanced MRI of the kidney for the differential diagnosis of localized renal lesions
}

\author{
Guenther Schneider $^{1} \cdot$ Thorsten Probst $^{1} \cdot$ Miles A. Kirchin $^{2} \cdot$ Jonas Stroeder $^{1}$ • \\ Peter Fries $^{1} \cdot$ Arno Buecker ${ }^{1}$
}

Received: 25 March 2015 / Accepted: 28 April 2015 / Published online: 19 June 2015

(C) The Author(s) 2015. This article is published with open access at Springerlink.com

\begin{abstract}
Objective To evaluate low-dose gadobenate dimeglumine-enhanced MRI for the differential diagnosis of malignant renal tumors.

Methods Sixty-two consecutive patients with unclear diagnosis at MDCT/ultrasound underwent dynamic CEMRI of the kidneys with $0.05 \mathrm{mmol} / \mathrm{kg}$ gadobenate dimeglumine. Retrospective image evaluation was performed by two blinded readers. Lesion diagnosis at CE-MRI was correlated with findings from histology following tumor resection or from imaging follow-up after at least 1 year. Assessments were performed of diagnostic quality and level of diagnostic information.

Results Thirty-nine $(63 \%)$ patients were correctly diagnosed with malignant lesions ( 36 with RCC, 2 with renal metastases, 1 with lymphoma) while $14(22.6 \%)$ patients were correctly diagnosed with benign $(n=12)$ or no $(n=2)$ lesions. Eight patients were considered false positive (5 with oncocytoma, 3 with atypical AML) and 1 patient false negative (atypical RCC). The sensitivity, specificity, accuracy, PPV, and NPV for the diagnosis of malignant renal lesions were $97.5 \%(39 / 40), 63.6 \%$ (14/22), $85.5 \%$ (53/62), $83.0 \%$ (39/47), and $93.3 \%$ (14/15), respectively. Images were excellent in 60 and good in 2
\end{abstract}

Guenther Schneider

dr.guenther.schneider@uks.eu

Miles A. Kirchin

miles.kirchin@bracco.com

1 Department of Diagnostic and Interventional Radiology, Saarland University Medical Center, 66421 Homburg/Saar, Germany

2 Global Medical and Regulatory Affairs, Bracco Imaging SpA, Milan, Italy patients. Minimal artifacts that did not compromise diagnosis were noted in $4 / 62$ patients.

Conclusion Low-dose gadobenate dimeglumineenhanced MRI is effective for the differential diagnosis of malignant renal tumors.

Keywords Gadobenate dimeglumine $\cdot$ Kidney $\cdot$ Renal MRI $\cdot$ Renal cell carcinoma

\section{Introduction}

Although renal cell carcinoma (RCC) is the most common malignant epithelial tumor of the kidney accounting for $85-90 \%$ of all solid renal tumors in adults and representing $5 \%$ of all cancers in men and $3 \%$ in women [1,2], benign solid neoplasms such as angiomyolipoma (AML) and oncocytoma represent $10-14 \%$ of all resected solid renal tumors [3-5]. A major clinical need therefore is to accurately differentiate RCC and other malignant renal lesions which typically require urgent surgical attention from benign solid neoplasms for which conservative management is usually indicated.

Whereas contrast-enhanced ultrasound (CEUS; [6-8]) and multi-detector-computed tomography (MDCT; [9-11]) are frequently first-line imaging techniques that reveal the presence of renal masses, often incidentally, subsequent work-up of patients with suspected malignant renal disease is usually performed using contrast-enhanced MR imaging (CE-MRI) because of the wide versatility of this technique and its ability to accurately identify fat within renal tumors. Several studies have demonstrated the effectiveness of CEMRI not only for the differential diagnosis of renal tumors but also for the accurate differentiation of different RCC sub-types [12-19]. 
To date, most recent protocols for CE-MRI of the kidney have utilized gadolinium-based contrast agents (GBCAs) at a dose of at least $0.1 \mathrm{mmol} / \mathrm{kg}$ bodyweight [13-18]. Moreover, the contrast agents used have invariably been conventional GBCAs such as gadopentetate dimeglumine which have standard $\mathrm{r} 1$ relaxivity values of approximately 4.2 $\mathrm{L} \mathrm{mmol}^{-1} \mathrm{~s}^{-1}$ at $1.5 \mathrm{~T}$ [20]. The aim of our study was to retrospectively determine the diagnostic performance of CE-MRI for the accurate diagnosis of malignant renal masses using a lower dose $(0.05 \mathrm{mmol} / \mathrm{kg} \mathrm{BW})$ of the much higher relaxivity GBCA gadobenate dimeglumine (MultiHance; Bracco Imaging SpA, Milan, Italy).

\section{Methods and materials}

\section{Patients}

Between January 2008 and December 2013, 184 consecutive patients at our institution underwent abdominal CEMRI with $0.05 \mathrm{mmol} / \mathrm{kg} \mathrm{BW}$ gadobenate dimeglumine with specific emphasis on dynamic CE-MRI of the kidneys. Of these 184 patients, $122(66.3 \%)$ underwent CE-MRI for follow-up of prior surgical resection of known malignant lesions or for routine-scheduled follow-up of known benign lesions. The remaining $62(33.7 \%)$ patients underwent CE-MRI because of equivocal renal tumors detected at multiphasic MDCT or ultrasound. This retrospective assessment focuses on these 62 patients. Institutional review board approval was obtained for the study. Written informed consent for the use of individual patient imaging data for research purposes was obtained from all patients.

The 62 evaluated patients included 37 men (mean age $58.5 \pm 16.1$ years; age range 10-79 years) and 25 women (mean age $62.5 \pm 11.9$ years; age range 37-80 years) with an overall mean age of $60.1 \pm 14.8$ years (age range 10-80 years).

\section{MR imaging}

MR imaging examinations were performed at 1.5 Tesla (Magnetom Vision, Sonata or Aera; Siemens Medical Systems, Erlangen, Germany). Patients were imaged in the supine position using a surface phased-array coil and the following sequences: (a) coronal T2-weighted halfFourier single-shot fast spin-echo [repetition time (TR) ms/echo time (TE) ms, 800-1100/60-92; section thickness, $5 \mathrm{~mm}$; gap, $1 \mathrm{~mm}$; matrix, $192 \times 256$; flip angle, $155^{\circ}-180^{\circ}$; field of view (FOV), $35-45 \mathrm{~cm}$ ], (b) axial dualecho T1-weighted in-phase and opposed-phase gradientecho (130-205/2.2-2.7, 4.5-5.2; flip angle, 70; section thickness, $5 \mathrm{~mm}$; gap, $1 \mathrm{~mm}$; matrix, $160 \times 256$; FOV,
$35-45 \mathrm{~cm})$. For contrast-enhanced imaging, a dynamic T1-weighted gradient-echo sequence (160-210/4.5-4.8; flip angle, $70^{\circ}$; slice thickness, 5-6 mm; gap, $1 \mathrm{~mm}$; matrix 154-180 × 256; FOV, 35-45 cm) was used when using a Siemens Magnetom Vision or Sonata. In the equilibrium phase, a T1w gradient-echo sequence with chemically selective fat saturation was acquired (134-160/2.3-2.7; flip angle, $70^{\circ}$; slice thickness, $5 \mathrm{~mm}$; gap, $1 \mathrm{~mm}$; matrix 154 $195 \times 256-320 ;$ FOV, 35-45 cm). With the Magnetom Aera system volume-interpolated breath-hold examinations (VIBE) were performed for both unenhanced and contrastenhanced imaging. A Dixon T1w sequence with the following parameters was acquired: TR $6.77 \mathrm{~ms}$; TE $2.38 \mathrm{~ms}$; slice thickness $3 \mathrm{~mm}$; FOV, $380 \mathrm{~mm}^{2}$; breath-hold acquisition time $21 \mathrm{~s}$, permitting acquisition of the following 4 T1w images at each slice level: in-phase image (standard T1), opposed-phase image, water-only image (fat suppressed T1), fat-only image.

Contrast-enhanced T1-weighted gradient-echo acquisitions were obtained dynamically in the cortico-medullary and nephrographic phases after administration of a bolus of $0.05 \mathrm{mmol} / \mathrm{kg}$ bodyweight of gadobenate dimeglumine at a rate of $2 \mathrm{~mL} / \mathrm{s}$ followed by a $20-\mathrm{mL}$ saline flush. For early examinations, the first pass was timed to the corticomedullary phase by best guess $(25 \mathrm{~s}$ post-start of contrast injection) while the nephrographic phase was acquired at approximately $5 \mathrm{~min}$ post-contrast injection. With the newer VIBE sequences, multiple phases were acquired and the appropriately timed sequences were chosen from the acquired data.

Routine diffusion-weighted imaging (DWI) for patients with suspected RCC was introduced into our department in September 2010 when the Magnetom Aera was installed. Of the 62 patients included in this assessment, DWI was performed for 29 patients using an echo planar imagingspin echo (EPI-SE) sequence with free breathing. The parameters for DWI acquisitions were as follows: TR/ $\mathrm{TE}[\mathrm{ms}]=6400 / 68$, section thickness, $6 \mathrm{~mm}$; gap, $0 \mathrm{~mm}$; number of slices, 35; matrix, $192 \times 156$; averages, 3; FOV $[\mathrm{mm} \times \mathrm{mm}], 380 \times 308$; spatial resolution $\left[\mathrm{mm}^{3}\right]$, $2.0 \times 2.0 \times 5.0 ; b$ values $\left[\mathrm{s} / \mathrm{mm}^{2}\right], 0,400,800 ;$ bandwidth [Hz/px], 1735; acquisition time [min], 4:30. Parallel imaging (GRAPPA 2) and fat suppression (SPAIR) were used.

\section{Image evaluation}

CE-MRI images were acquired as part of routine clinical practice. Based on CE-MRI diagnosis in conjunction with available findings from prior diagnostic imaging studies, patients were referred either for surgical resection of the detected lesion(s) or for conservative management. Typically, lesions that show no evidence of homogeneous fat 
distribution on unenhanced MR images but which demonstrate contrast enhancement following contrast administration are malignant in nature. Occasionally, RCC may present with minimal fat in just one area of the lesion. For the purposes of this study, lesions that demonstrated either of these features were considered malignant. Final lesion diagnosis was based on the histopathologic results for the specimen obtained at surgical resection or on imaging follow-up obtained after at least 1 year.

Subsequent retrospective evaluation of images was performed qualitatively by two readers $(\mathrm{PF}, \mathrm{AB} ; 8$ and 15 years' experience in abdominal MRI, respectively) in consensus who were not involved in the conduct of the studies and who were fully blinded to the clinical history of the patients, the results of all diagnostic imaging examinations, and to the final clinical diagnosis. Assessments were performed in terms of quality of kidney visualization (insufficient, poor, moderate, good, excellent), presence of artifacts (severe, moderate, minimal, none), extent of diagnostic information (unsatisfactory, partial, satisfactory, complete), and overall diagnostic value (limited, satisfactory, high). Additional assessments were performed of lesion size and of any additional diagnostic information.

\section{Statistical analysis}

Diagnostic performance for the characterization of lesions as malignant or benign was performed at the patient level for all 62 patients using CE-MRI images plus unenhanced axial dual-echo T1-weighted in-phase and opposed-phase gradient-echo images for the analysis of fat content. For this evaluation, patients diagnosed with malignant renal lesions at CE-MRI which were confirmed as malignant at final diagnosis were considered true positive (TP) while patients diagnosed with benign renal lesions or no lesions at CE-MRI which were confirmed as benign or absent at final diagnosis were considered true negative (TN). Patients with renal lesions diagnosed as malignant at CE-MRI which were confirmed as benign at final diagnosis were considered false positive (FP) while patients with renal lesions diagnosed as benign at CE-MRI which were confirmed as malignant at final diagnosis were considered false negative (FN). Based on these findings, determinations were made of the sensitivity, specificity, accuracy, positive predictive value (PPV), and negative predictive value (NPV) of CEMRI with $0.05 \mathrm{mmol} / \mathrm{kg}$ gadobenate dimeglumine for the diagnosis of malignant renal tumors.

\section{Results}

Based on CE-MRI findings alone, 39/62 (63 \%) patients were diagnosed with malignant renal lesions. These
39 patients included 29 with RCC confirmed at histology (Figs. 1, 2), 7 patients with RCC confirmed at imaging follow-up, 2 patients with renal metastases confirmed at histology (in patients with primary lung cancer and breast cancer), and 1 patient with lymphoma confirmed at imaging follow-up. The 29 RCC confirmed at histology included 20 clear cell tumors, 6 papillary tumors, 1 mixed clear cell/papillary tumor, and 2 chromophobe tumors. These 39 patients were considered TP for renal cancer. A further 14/62 (22.6\%) patients were diagnosed with benign $(n=12)$ or no $(n=2)$ renal lesions. The 12 patients with benign lesions included 8 with complicated renal cysts confirmed at imaging follow-up $(n=7)$ or histology $(n=1)$, 3 patients with AML confirmed at imaging follow-up [including one 10-year-old boy with multiple bilateral AML (Fig. 3), one of which was resected due to its rapid growth and risk of hemorrhage], and 1 patient diagnosed with inflammatory tumor (Fig. 4) which was confirmed at imaging follow-up (pyelonephritis with renal abscess formation, which completely resolved under antibiotic therapy). These 14 patients were considered $\mathrm{TN}$ for malignant renal tumors. The remaining 9 patients included 8 patients that were diagnosed with malignant lesions $(n=7)$ or an unspecified lesion $(n=1)$ at CE-MRI which were confirmed as benign [oncocytoma in 5 patients (Fig. 5), atypical AML in 3 patients] at either histology $(n=6)$ or imaging follow-up $(n=2)$. One of these patients was diagnosed with both an RCC and AML which were confirmed as two AML at histology. These 8 patients were considered FP for malignant renal tumors. The final patient was considered to have an unspecific lesion at CE-MRI which was confirmed as an atypical RCC at histology. Based on its appearance at CE-MRI (solid, hypovascular, showing almost no contrast uptake, and without characteristic features of a benign lesion), the lesion was recommended for resection. Nevertheless, for the purposes of this study, since a malignant diagnosis was not made at CE-MRI, this patient was considered FN for malignant renal tumors.

The overall mean size of lesions was $27.1 \pm 20.6 \mathrm{~mm}$ (range: 4-94 mm). The overall mean size of lesions diagnosed as malignant $(27.6 \pm 21.6 \mathrm{~mm}$; range: $4-94 \mathrm{~mm})$ was slightly larger than those diagnosed as benign $(17.3 \pm 16.2 \mathrm{~mm}$; range: $4-67 \mathrm{~mm})$.

Based on a total of $39 \mathrm{TP}$ patients, $14 \mathrm{TN}$ patients, $8 \mathrm{FP}$ patients, and $1 \mathrm{FN}$ patient, the overall sensitivity, specificity, accuracy, PPV, and NPV were determined to be $97.5 \%$ (39/40), $63.6 \%$ (14/22), $85.5 \%$ (53/62), $83.0 \%$ (39/47), and $93.3 \%(14 / 15)$, respectively, for the diagnosis of renal cancer on CE-MRI with $0.05 \mathrm{mmol} / \mathrm{kg}$ gadobenate dimeglumine. No gender-based differences were apparent: the sensitivity, specificity, accuracy, PPV, and NPV for male patients [95.6\% (22/23), $71.4 \%$ (10/14), $86.5 \%$ (32/37), $84.6 \%(22 / 26)$, and $90.9 \%(10 / 11)]$ were similar to that of 

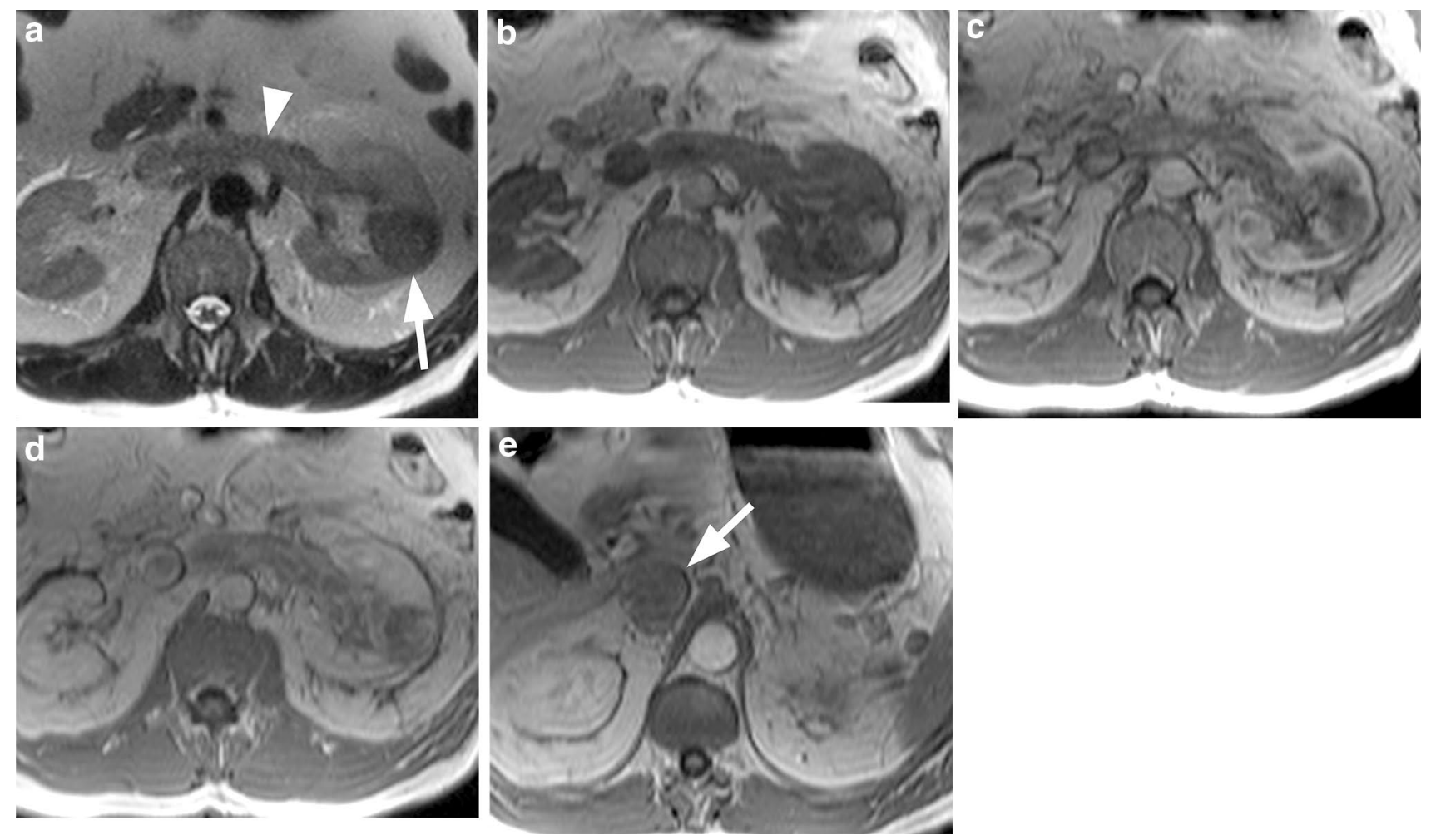

Fig. 1 Renal cell carcinoma with a tumor thrombus in the left renal vein and IVC. The T2w HASTE sequence (a) reveals a small lesion in the left kidney (arrow) together with enlargement of the left renal vein, filled with material that is isointense to the normal renal parenchyma (arrowhead). On the corresponding T1w image (b) the tumor is hyperintense and no flow void is visible within the left renal vein. After injection of gadobenate dimeglumine at a dose of $0.05 \mathrm{mmol} / \mathrm{kg}$

female patients [100\% (17/17), $50 \%(4 / 8), 84 \%(21 / 25)$, $80.9 \%(17 / 21)$, and $100 \%(4 / 4)]$.

Among the 37 patients with confirmed RCC (36 TP patients plus $1 \mathrm{FN}$ patient), additional findings in the liver were noted in 6 patients [metastases in 3 patients (Fig. 6), hemangiomas in 3 patients].

\section{Blinded qualitative evaluation}

The quality of kidney visualization was rated as excellent in 60 patients and as good in 2 patients. In no patient was the quality of visualization considered insufficient, poor, or moderate. Images were completely free of artifacts in 58 patients; in the remaining 4 patients, minimal artifacts were noted which did not compromise image interpretation or diagnosis. The necessary imaging information needed to make a diagnosis was considered complete in all 62 patients; the value of this diagnostic information was considered high in 61 patients and satisfactory in just one patient.
BW the tumor and left renal vein show inhomogeneous enhancement in the early phase (c) with early contrast wash-out in the later phase (d). This enhancement suggests a RCC with a tumor thrombus in the left renal vein. Note the complete occlusion of the IVC (e), with the IVC completely filled with solid tumor material from the tumor thrombus (arrow in $\mathbf{e}$ )

\section{Discussion}

An early prospective assessment of the diagnostic performance of CE-MRI for the differentiation of patients with malignant renal lesions from patients with benign renal lesions determined values for sensitivity, specificity, PPV, and NPV of $93.8 \%$ (15/16), $66.7 \%$ (8/12), $78.9 \%(15 / 19)$, and $88.8 \%(8 / 9)$, respectively [9]. In that study, all oncocytomas were falsely classified as carcinomas (FP lesions) while three AMLs and three inflammatory lesions were correctly classified as benign (TN lesions) resulting in an overall per-patient-based accuracy of $82.1 \%$ (23/28). In common with other studies focused on the CE-MRI evaluation of renal masses [13-18], the study utilized a conventional GBCA at a dose of $0.1 \mathrm{mmol} / \mathrm{kg}$ bodyweight [9]. Our retrospective study, performed as part of routine clinical practice in patients referred for abdominal/renal MRI because of unclear findings on multiphasic MDCT, utilized gadobenate dimeglumine at a dose of only $0.05 \mathrm{mmol} / \mathrm{kg}$ bodyweight. Our values for sensitivity, specificity, accuracy, 


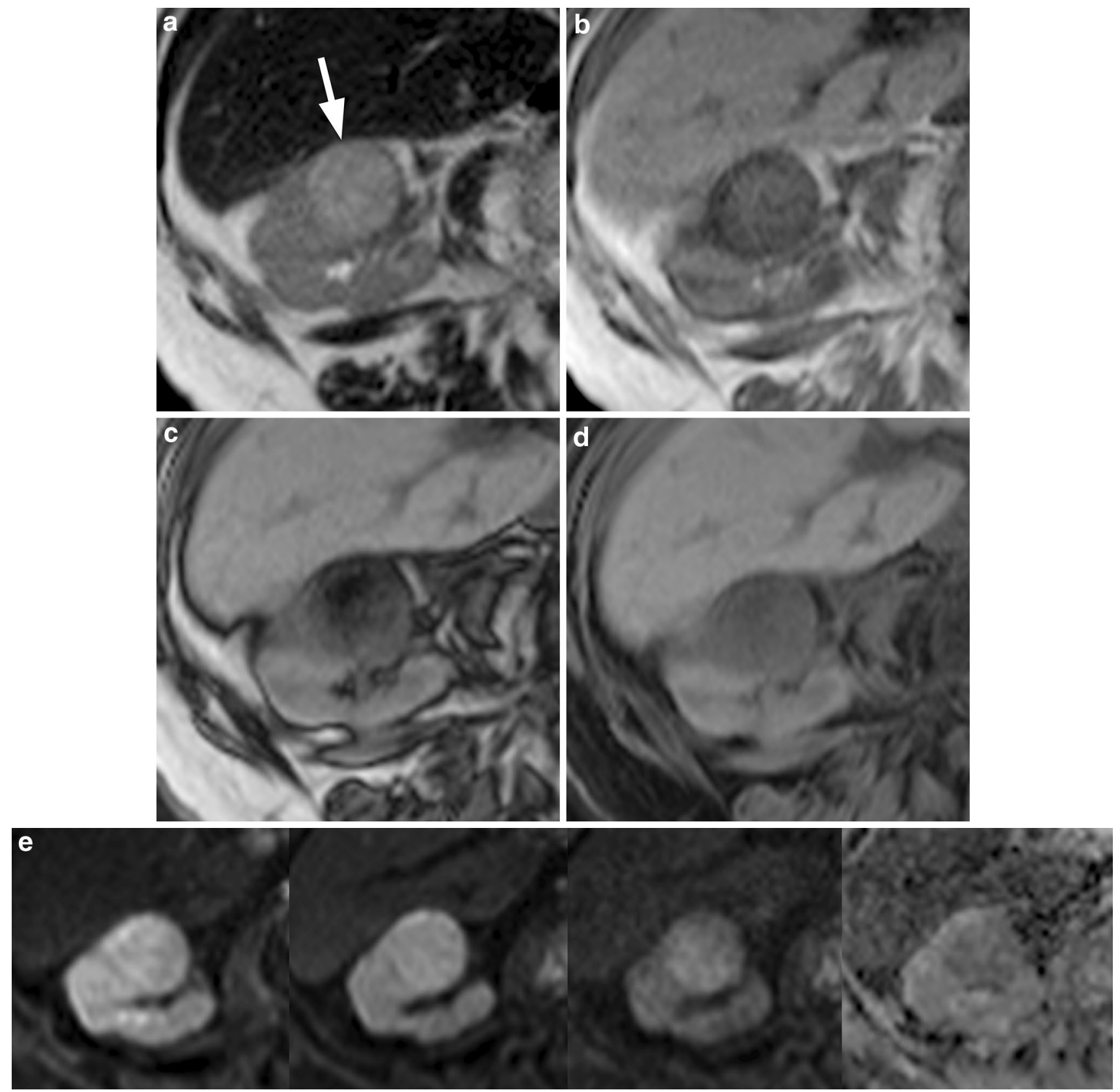

Fig. 2 Renal cell carcinoma. On the T2w HASTE sequence (a) a hyperintense lesion (arrow) is visible in the right kidney. On the VIBE sequence with acquisition of in-phase, opposed-phase, and Dixon fs-images (b-d) in one single breath-hold, the lesion demonstrates a hypointense rim on the in-phase image (b), some hypointense areas in the center on the opposed-phase image (c) indicating fat within the tumor, and a homogeneous hypointense signal in the

PPV, and NPV of $97.5 \%$ (39/40), $63.6 \%$ (14/22), $85.5 \%$ $(53 / 62), 83.0 \%(39 / 47)$, and $93.3 \%(14 / 15)$, respectively, bear excellent comparison with those of the above study [9]. Although comparison with a full dose of another GBCA was not performed in our study, our results suggest that equivalent diagnostic performance might be achieved fat suppressed image (d). The diffusion-weighted images and the ADC-map $(\mathbf{e} ; b=50,400,800+$ ADC from left to right) reveal restricted diffusion and a hypointense appearance of the tumor in the ADC-map. Dynamic contrast-enhanced fs VIBE-sequences $(\mathbf{f}-\mathbf{k})$ ( $0.05 \mathrm{mmol} / \mathrm{kg}$ gadobenate dimeglumine) demonstrate tumor hypervascularity with early contrast wash-out. All findings indicate a RCC which was proven on histology after partial nephrectomy

with just half the amount of gadolinium if gadobenate dimeglumine is the utilized GBCA. In this regard, a previous intra-individual crossover comparison of $0.05 \mathrm{mmol} / \mathrm{kg}$ gadobenate dimeglumine with $0.1 \mathrm{mmol} / \mathrm{kg}$ gadopentetate dimeglumine in patients undergoing CE-MRI of the liver revealed clear equivalence during the dynamic phase of 

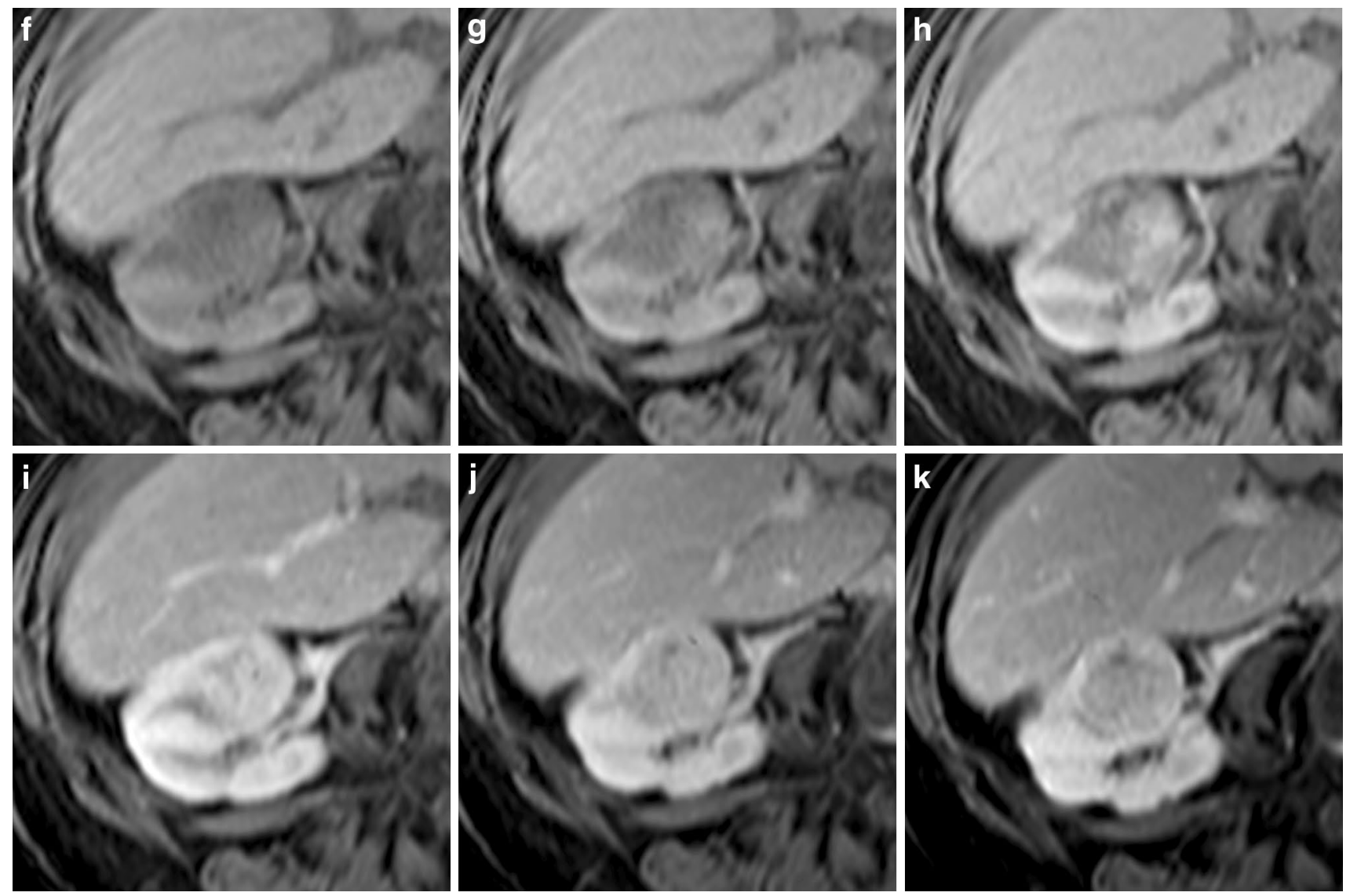

Fig. 2 continued

contrast enhancement with significant superiority for gadobenate dimeglumine during the delayed hepatobiliary phase [21].

Clearly, these findings are potentially very important given the risk of nephrogenic systemic fibrosis (NSF) in patients with severe renal insufficiency given the possibility of compromised renal function in patients with renal tumors and the fact that new-onset post-operative chronic kidney disease (CKD) is a relatively frequent occurrence in patients undergoing curative surgery for renal cell carcinoma, particularly in patients aged 60 years or older and in patients with already decreased preoperative renal function [22-26]. In this regard, although symptoms of NSF typically manifest within approximately 3 months of GBCA administration, longer lead-times are not unknown [27, 28]. Notably, no unconfounded cases of NSF have yet been reported for gadobenate dimeglumine despite its regular use in patients at heightened risk of developing this disease [29-32]. As reported elsewhere for a variety of MR indications [21, 33-40], the possibility to use a reduced dose of gadobenate dimeglumine reflects the higher $\mathrm{r} 1$ relaxivity of this agent (6.2 vs. $3.9-4.6 \mathrm{~L} \mathrm{mmol}^{-1} \mathrm{~s}^{-1}$ at $1.5 \mathrm{~T}$ according to recent data [20]) which derives from weak, transient interaction of the Gd-BOPTA contrast-effective molecule with serum albumin $[41,42]$ and a resulting slowing of the molecular tumbling rate which leads to greater shortening of the T1 relaxation time and thus greater signal enhancement at equivalent dose [43].

Unlike conventional GBCAs which are excreted almost exclusively via the kidneys, approximately $2-4 \%$ of the injected dose of gadobenate dimeglumine is eliminated via the hepatobiliary pathway $[44,45]$. Although this level of hepatobiliary excretion does not alter the pharmacokinetic profile of gadobenate dimeglumine relative to the profiles of conventional GBCAs [44, 45] or the characteristic dynamic enhancement patterns of frequently encountered tumors in the liver [46-51] and breast [52-55], it is sufficient to permit delayed hepatobiliary phase imaging of the liver for the improved detection and characterization of liver lesions [46-51]. On the one hand, the similar dynamic enhancement behavior of gadobenate dimeglumine relative to conventional GBCAs, even at half the dose [21], may explain the similar diagnostic performance of gadobenate dimeglumine for renal MRI not only in 

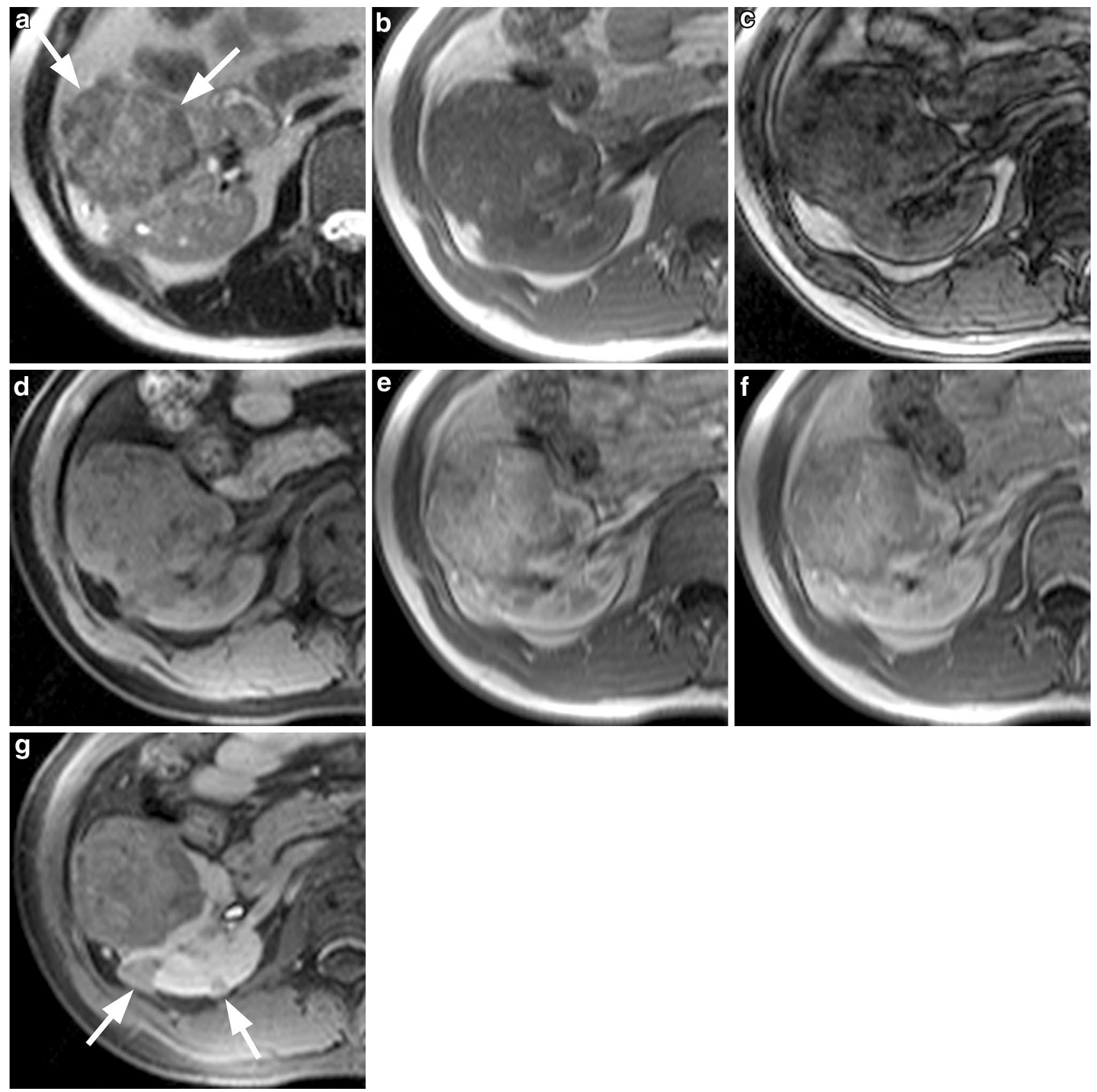

Fig. 3 Multiple angiomyolipoma. The T2w HASTE sequence (a) shows a large mass (arrow) in the right kidney with heterogeneously distributed fat throughout. On the corresponding T1w image (b) the tumor shows areas of increased signal intensity, indicating either fat or hemorrhage within the tumor. The areas of increased signal on the T1w image are hypointense on the opposed-phase image (c) and the T1w fs image (d), indicating fat within the tumor. After injection of $0.05 \mathrm{mmol} / \mathrm{kg}$ gadobenate dimeglumine, the lesion shows inhomoge-

terms of the accurate detection of malignant lesions but also the accurate differentiation of malignant from benign lesions. In this regard, the FP lesions detected in our study neous but strong vascularization in the early arterial phase (e) with early wash-out in the later phase (f). In the fat suppressed image in the equilibrium phase (g) additional lesions (arrows) are visible that are hypointense compared with the normal renal parenchyma. Taken together these findings indicate multiple angiomyolipoma, which were proven after nephron sparing surgery of the largest lesion (performed because of the risk of bleeding)

(5 oncocytoma, 3 atypical AML i.e., AML without visible fat) are typical of the FP lesions detected elsewhere $[9,15,17-19,56,57]$. Both of these benign lesions share 

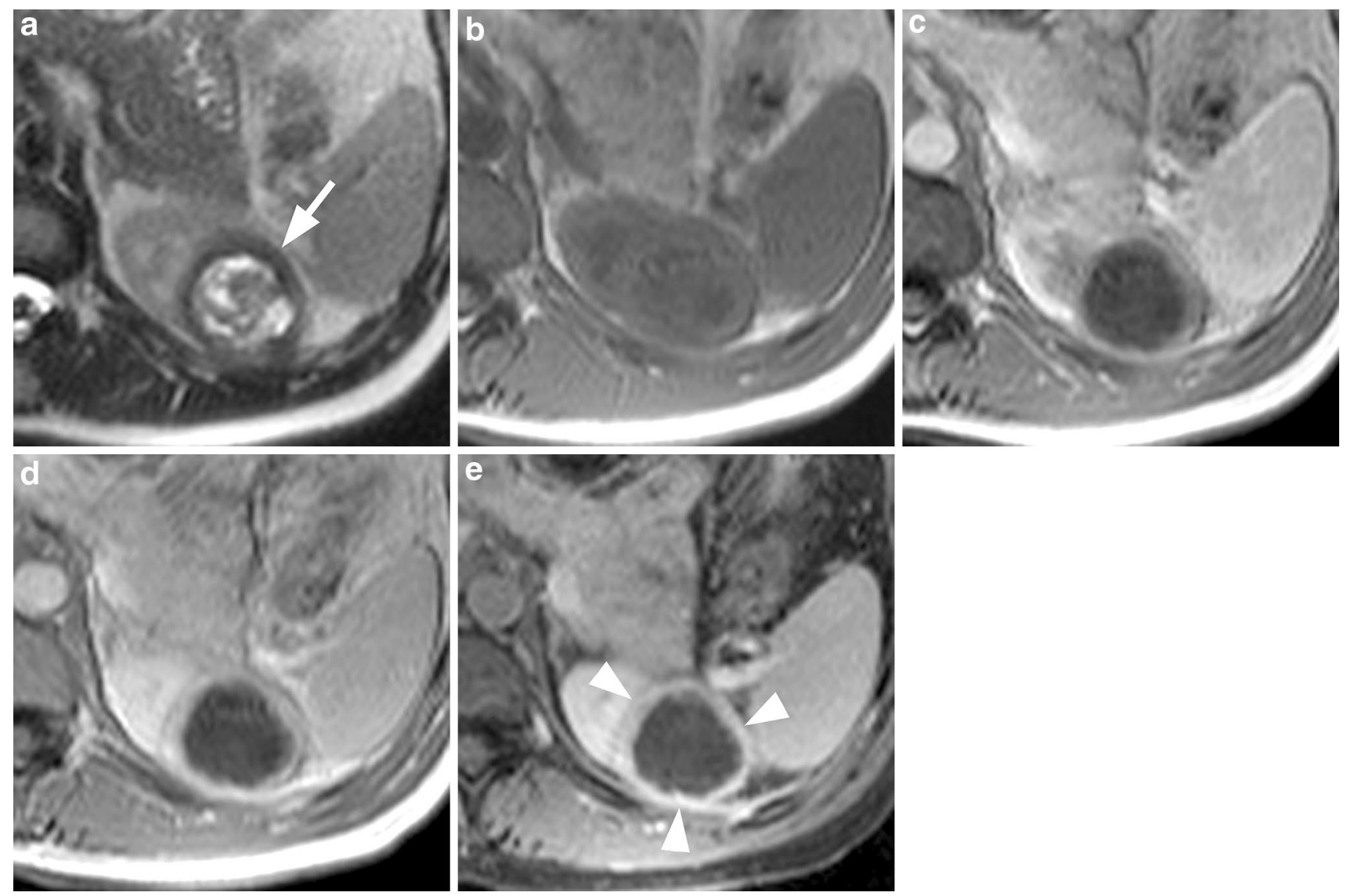

Fig. 4 Renal abscess. The T2w HASTE sequence (a) shows an inhomogeneous cystic lesion (arrow) of the upper pole of the left kidney surrounded by a hypointense rim. The lesion is almost isointense to the renal cortex and medulla on the unenhanced T1w image (b). After injection of $0.05 \mathrm{mmol} / \mathrm{kg}$ gadobenate dimeglumine the rim surrounding the lesion shows, compared with the renal cortex, delayed but homogeneous contrast uptake $(\mathbf{c}, \mathbf{d})$, whereas the central areas remain hypointense without contrast enhancement. The T1w fs image during the equilibrium phase (e) shows persistent enhancement of the rim (arrowheads), indicating a wall of inflammatory tissue surrounding an abscess formation

administration, particularly given that as many as $30 \%$ of patients with RCC present with metastases [60-62] and that metastatic RCC has a relatively poor prognosis (5-year survival rate approximately $20 \%[63,64])$. Several studies have shown that hepatobiliary phase imaging with gadobenate dimeglumine improves the detection of hepatic metastases relative to that achievable with other techniques [65-67]. A further 3 patients with RCC in our study had liver hemangiomas detected incidentally during dynamic phase imaging. Characterization of these lesions and differentiation from liver metastases was correctly achieved in all cases reflecting the suitability of gadobenate dimeglumine for liver imaging. In this regard, gadobenate dimeglumine is specifically approved in Europe and elsewhere for MR imaging of the liver at a dose of $0.05 \mathrm{mmol} / \mathrm{kg}$ bodyweight [68]. As noted elsewhere $[29,31,69]$, the partial hepatobiliary elimination of gadobenate dimeglumine combined with the 



Fig. 5 Multiple oncocytoma. The T2w HASTE sequence (a) shows two partially isointense and partially hyperintense lesions (arrows) at the upper pole of the left kidney. On the corresponding T1w image (b) the lesions are almost isointense to the renal cortex. After injection of $0.05 \mathrm{mmol} / \mathrm{kg}$ gadobenate dimeglumine, the lesions show an inhomogeneous hyervascularization in an early arterial phase (c) with homogenous contrast uptake in the later phase (d). On the T1w fs image (e) in the equilibrium phase the lesions show inhomogeneous contrast uptake. Diagnosis based on CE-MRI was multiple RCC; however, histology confirmed the lesions to be multiple oncocytoma administration of a reduced dose in at-risk patients may in part explain the absence of NSF in patients given this GBCA.

Our study has several limitations. First, it was a retrospective assessment of consecutive patients rather than a prospective evaluation. However, it should be noted that our findings for diagnostic performance were similar to those obtained elsewhere in a smaller prospective study [9]. Second, the evaluation of image quality was subjective rather than objective based on pre-defined quantitative or qualitative measures of enhancement. Finally, DWI was performed in only 29 patients in our study. Numerous studies have demonstrated the value of DWI for the improved characterization of detected renal neoplasms [15, 16, 57, 70-74]. One such study [15] showed that the addition of ADC information to CE-MRI findings led to the reclassification of 3 of 6 oncocytomas and 2 of 2 multilocular cystic nephromas that had originally been misdiagnosed as malignant on CE-MRI alone, thereby increasing specificity from 89 to $96 \%$. The use of gadobenate dimeglumine for DWI has previously been demonstrated in 83 patients with 85 renal masses [75]. In that study, a dose of $0.1 \mathrm{mmol} / \mathrm{kg}$ gadobenate dimeglumine enabled sensitive and specific differentiation of clear cell, papillary, and chromophobic RCC. Specifically, the sensitivity and specificity values for the differentiation of clear cell from non-clear cell RCC were high (95.9 and $94.4 \%$, respectively), which is particularly important given that patients with chromophobic or papillary RCC (i.e., not clear cell RCC) have a better prognosis than patients with clear cell RCC [76] and that these subtypes respond differently to molecularly targeted therapies.

In conclusion, our study confirms that gadobenate dimeglumine at a dose of $0.05 \mathrm{mmol} / \mathrm{kg}$ bodyweight is 

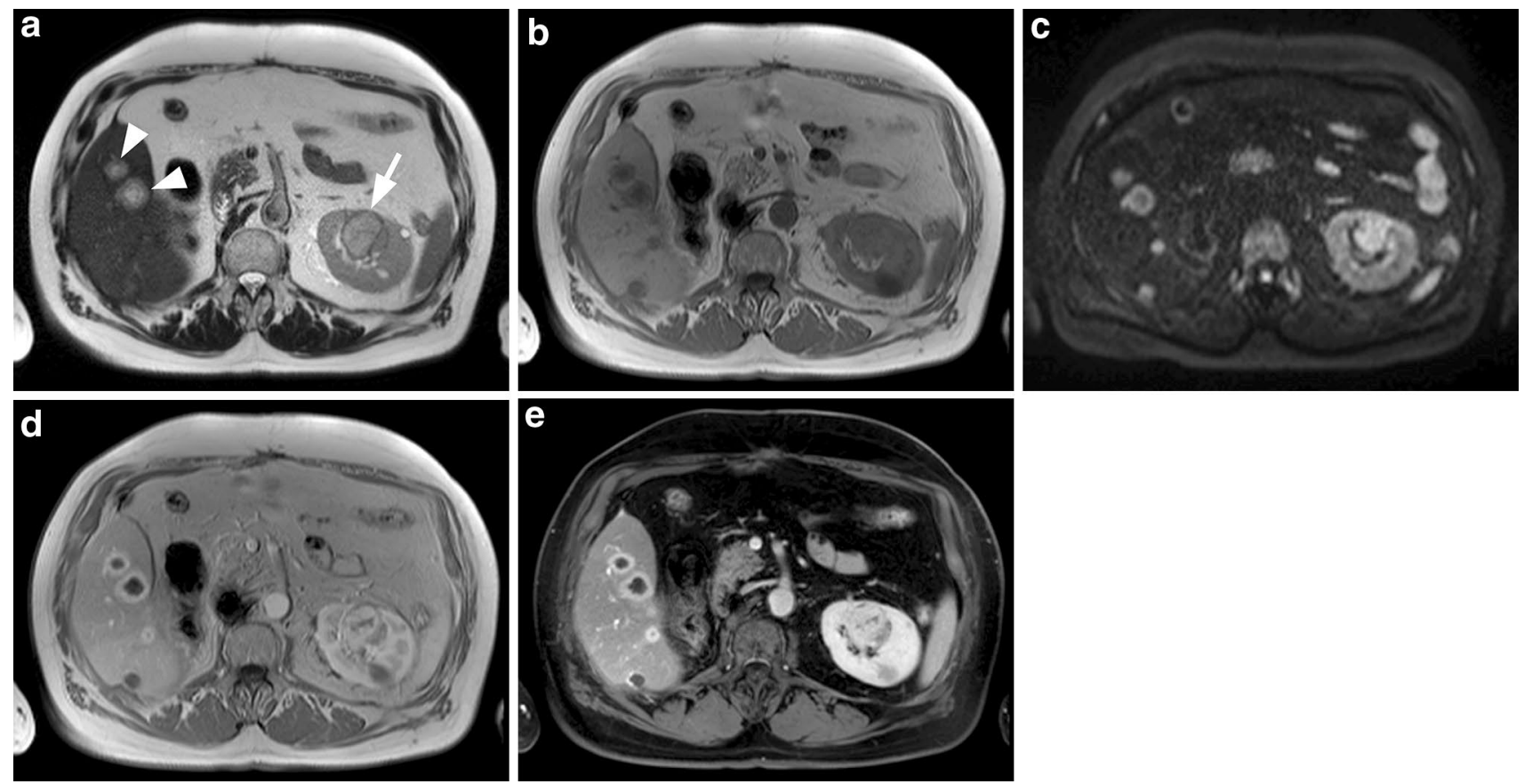

Fig. 6 RCC with multiple liver metastases. The T2w HASTE sequence (a) reveals multiple liver metastases (arrowheads) from a RCC of the left kidney (arrow). On the corresponding T1w image (b) the liver lesions are hypointense to the liver parenchyma, whereas the $\mathrm{RCC}$ is isointense with the renal cortex. On the DWI image with a $b$-value of 800 (c) the liver metastases as well as the tumor in the left

appropriate for MR imaging of the kidney, for the detection and differential diagnosis of renal neoplasms with high diagnostic performance.

Conflict of interest Dr. Guenther Schneider has received a research grant and speaker honoraria from Bracco Imaging SpA. Dr. Miles A. Kirchin is an employee of Bracco Imaging SpA. The other authors declare that they have no conflict of interest.

Ethical standards This article does not contain any studies with human participants or animals performed by any of the authors.

Human/animal rights This was a retrospective study. For this type of study formal consent is not required. This article does not contain any studies with animals performed by any of the authors.

Informed consent Informed consent for the possible future elaboration of data was obtained from all individual participants included in the study at the time of the initial clinical examination.

Open Access This article is distributed under the terms of the Creative Commons Attribution 4.0 International License (http://creativecommons.org/licenses/by/4.0/), which permits unrestricted use, distribution, and reproduction in any medium, provided you give appropriate credit to the original author(s) and the source, provide a link to the Creative Commons license, and indicate if changes were made. kidney are hyperintense indicating diffusion restriction. After injection of $0.05 \mathrm{mmol} / \mathrm{kg}$ gadobenate dimeglumine, the liver lesions as well as the RCC of the left kidney are hypervascular during the early arterial phase (d) with the RCC showing early contrast wash-out on the $\mathrm{T} 1 \mathrm{w}$ fs image (e) during a later phase

\section{References}

1. Siegel R, Ma J, Zou Z, Jemal A (2014) Cancer statistics, 2014. CA Cancer J Clin 64:9-29

2. Pantuck AJ, Zisman A, Belldegrun AS (2001) The changing natural history of RCC. J Urol 166:1611-1623

3. Frank I, Blute ML, Cheville JC, Lohse CM, Weaver AL, Zincke $\mathrm{H}$ (2003) Solid renal tumors: an analysis of pathological features related to tumor size. J Urol 170:2217-2220

4. Fujii Y, Komai Y, Saito K et al (2008) Incidence of benign pathologic lesions at partial nephrectomy for presumed RCC renal masses: Japanese dual-center experience with 176 consecutive patients. Urology 72:598-602

5. Kutikov A, Fossett LK, Ramchandani P et al (2006) Incidence of benign pathologic findings at partial nephrectomy for solitary renal mass presumed to be renal cell carcinoma on preoperative imaging. Urology 68:737-740

6. Meola M, Petrucci I, Giovannini L, Colombini E, Villa A (2012) Ultrasound and color Doppler imaging for kidney and urinary tract tumors. G Ital Nefrol 29:452-466

7. Houtzager S, Wijkstra H, de la Rosette JJ, Laguna MP (2013) Evaluation of renal masses with contrast-enhanced ultrasound. Curr Urol Rep 14:116-123

8. Wang C, Yu C, Yang F, Yang G (2014) Diagnostic accuracy of contrast-enhanced ultrasound for renal cell carcinoma: a metaanalysis. Tumour Biol. (Epub ahead of print)

9. Beer AJ, Dobritz M, Zantl N, Weirich G, Stollfuss J, Rummeny EJ (2006) Comparison of 16-MDCT and MRI for characterization of kidney lesions. AJR Am J Roentgenol 186:1639-1650 
10. Millet I, Doyon FC, Hoa D et al (2011) Characterization of small solid renal lesions: can benign and malignant tumors be differentiated with CT? AJR Am J Roentgenol 197:887-896

11. Kim MH, Lee J, Cho G, Cho KS, Kim J, Kim JK (2013) MDCT-based scoring system for differentiating angiomyolipoma with minimal fat from renal cell carcinoma. Acta Radiol 54:1201-1209

12. Verswijvel G, Oyen R (2004) Magnetic resonance imaging in the detection and characterization of renal diseases. Saudi J Kidney Dis Transpl 15:283-299

13. Tello R, Davison BD, O'Malley M et al (2000) MR imaging of renal masses interpreted on CT to be suspicious. AJR Am J Roentgenol 174:1017-1022

14. Hecht EM, Israel GM, Krinsky GA et al (2004) Renal masses: quantitative analysis of enhancement with signal intensity measurements versus qualitative analysis of enhancement with image subtraction for diagnosing malignancy at MR imaging. Radiology 232:373-378

15. Taouli B, Thakur RK, Mannelli L et al (2009) Renal lesions: characterization with diffusion-weighted imaging versus contrast-enhanced MR imaging. Radiology 251:398-407

16. Kim S, Jain M, Harris AB et al (2009) T1 hyperintense renal lesions: characterization with diffusion-weighted MR imaging versus contrast-enhanced MR imaging. Radiology 251:796-807

17. Sun MR, Ngo L, Genega EM et al (2009) Renal cell carcinoma: dynamic contrast-enhanced MR imaging for differentiation of tumor subtypes-correlation with pathologic findings. Radiology 250:793-802

18. Sasiwimonphan K, Takahashi N, Leibovich BC, Carter RE, Atwell TD, Kawashima A (2012) Small $(<4 \mathrm{~cm})$ renal mass: differentiation of angiomyolipoma without visible fat from renal cell carcinoma utilizing MR imaging. Radiology 263:160-168

19. Gurel S, Narra V, Elsayes KM, Siegel CL, Chen ZE, Brown JJ (2013) Subtypes of renal cell carcinoma: MRI and pathological features. Diagn Interv Radiol 19:304-311

20. Shen Y, Goerner FL, Snyder C et al (2015) T1 relaxivities of gadolinium-based magnetic resonance contrast agents in human whole blood at 1.5, 3, and 7 T. Invest Radiol 50:330-338

21. Schneider G, Maas R, Schultze Kool L et al (2003) Low-dose gadobenate dimeglumine versus standard dose gadopentetate dimeglumine for contrast-enhanced magnetic resonance imaging of the liver: an intra-individual crossover comparison. Invest Radiol 38:85-94

22. Kong HJ, Park JS, Kim DY, Shin HS, Jung HJ (2013) Renal function following curative surgery for renal cell carcinoma: who is at risk for renal insufficiency? Korean J Urol 54:830-833

23. Kim SH, Lee SE, Hong SK et al (2013) Incidence and risk factors of chronic kidney disease in Korean patients with tla renal cell carcinoma before and after radical or partial nephrectomy. Jpn J Clin Oncol 43:1243-1248

24. Fergany A (2013) Chronic renal insufficiency after partial nephrectomy for T1b tumors. Curr Opin Urol 23:394-398

25. Shin S, Han Y, Park H et al (2013) Risk factors for acute kidney injury after radical nephrectomy and inferior vena cava thrombectomy for renal cell carcinoma. J Vasc Surg 58:1021-1027

26. Jeon HG, Choo SH, Sung HH et al (2014) Small tumour size is associated with new-onset chronic kidney disease after radical nephrectomy in patients with renal cell carcinoma. Eur J Cancer 50:64-69

27. Collidge TA, Thomson PC, Mark PB et al (2007) Gadoliniumenhanced MR imaging and nephrogenic systemic fibrosis: retrospective study of a renal replacement therapy cohort. Radiology 245:168-175
28. Thomson LK, Thomson PC, Kingsmore DB et al (2015) Diagnosing nephrogenic systemic fibrosis in the post-FDA restriction era. J Magn Reson Imag 41:1268-1271

29. Spinazzi A (2014) MRI contrast agents and nephrogenic systemic fibrosis. In: Shellock FG, Crues JV (eds) MRI bioeffects, safety, and patient management. Biomedical Research Publishing Group, Los Angeles, pp 256-281

30. Heverhagen JT, Krombach GA, Gizewski E (2014) Application of extracellular gadolinium-based MRI contrast agents and the risk of nephrogenic systemic fibrosis. Rofo 186:661-669

31. Nandwana SB, Moreno CC, Osipow MT, Sekhar A, Cox KL (2015) Gadobenate dimeglumine administration and nephrogenic systemic fibrosis: is there a real risk in patients with impaired renal function? Radiology 15:142423. (Epub ahead of print)

32. Soulez G, Bloomgarden DC, Rofsky NM, et al (2015) Nephrogenic systemic fibrosis in patients with stages 3 to 5 chronic kidney disease undergoing MRI with the injection of gadobenate dimeglumine or gadoteridol: findings from prospective cohort studies. Am J Roengenol. (in press)

33. Balci NC, Inan N, Anik Y, Erturk MS, Ural D, Demirci A (2006) Low-dose gadobenate dimeglumine versus standard-dose gadopentate dimeglumine for delayed contrast-enhanced cardiac magnetic resonance imaging. Acad Radiol 13:833-839

34. Bauner KU, Reiser MF, Huber AM (2009) Low dose gadobenate dimeglumine for imaging of chronic myocardial infarction in comparison with standard dose gadopentetate dimeglumine. Invest Radiol 44:95-104

35. Pediconi F, Fraioli F, Catalano C et al (2003) Gadobenate dimeglumine (Gd-BOPTA) vs gadopentetate dimeglumine (Gd-DTPA) for contrast-enhanced magnetic resonance angiography (MRA): improvement in intravascular signal intensity and contrast to noise ratio. Radiol Med (Torino) 106:87-93

36. Prokop M, Schneider G, Vanzulli A et al (2005) Contrastenhanced MR angiography of the renal arteries: blinded multicenter crossover comparison of gadobenate dimeglumine and gadopentetate dimeglumine. Radiology 234:399-408

37. Li Y, Li X, Li D et al (2013) Multicenter, intraindividual comparison of single-dose gadobenate dimeglumine and double-dose gadopentetate dimeglumine for MR angiography of the supraaortic arteries (the supra-aortic value study). AJNR Am J Neuroradiol 34:847-854

38. Wang J, Yan F, Liu J et al (2013) Multicenter, intra-individual comparison of single dose gadobenate dimeglumine and double dose gadopentetate dimeglumine for MR Angiography of the peripheral arteries (the peripheral VALUE study). J Magn Reson Imag 38:926-937

39. Woodard PK, Chenevert TL, Sostman HD et al (2012) Signal quality of single dose gadobenate dimeglumine pulmonary MRA examinations exceeds quality of MRA performed with double dose gadopentetate dimeglumine. Int J Cardiovasc Imaging 28:295-301

40. Khouri Chalouhi K, Papini GD, Bandirali M, Sconfienza LM, Di Leo G, Sardanelli F (2014) Less is better? Intraindividual and interindividual comparison between $0.075 \mathrm{mmol} / \mathrm{kg}$ of gadobenate dimeglumine and $0.1 \mathrm{mmol} / \mathrm{kg}$ of gadoterate meglumine for cranial MRI. Eur J Radiol 83:1245-1249

41. Cavagna FM, Maggioni F, Castelli PM et al (1997) Gadolinium chelates with weak binding to serum proteins. A new class of high-efficiency, general purpose contrast agents for magnetic resonance imaging. Invest Radiol 32:780-796

42. Giesel FL, von Tengg-Kobligk H, Wilkinson ID et al (2006) Influence of human serum albumin on longitudinal and transverse relaxation rates (R1 and R2) of magnetic resonance contrast agents. Invest Radiol 41:222-228 
43. Kanal E, Maravilla K, Rowley HA (2014) Gadolinium contrast agents for CNS imaging: current concepts and clinical evidence. AJNR Am J Neuroradiol 35:2215-2226

44. Spinazzi A, Lorusso V, Pirovano G, Taroni P, Kirchin M, Davies A (1998) MultiHance clinical pharmacology: biodistribution and MR enhancement of the liver. Acad Radiol 5:S86-S89

45. Spinazzi A, Lorusso V, Pirovano GP, Kirchin M (1999) Safety, tolerability, biodistribution and MR enhancement of the liver with Gd-BOPTA: results of clinical pharmacology and pilot imaging studies in non-patient and patient volunteers. Acad Radiol 6:282-291

46. Pirovano G, Vanzulli A, Marti-Bonmati L et al (2000) Evaluation of the accuracy of gadobenate dimeglumine-enhanced MR imaging in the detection and characterization of focal liver lesions. AJR Am J Roentgenol 175:1111-1120

47. Grazioli L, Morana G, Federle MP et al (2001) Focal nodular hyperplasia: morphological and functional information from MR imaging with gadobenate dimeglumine. Radiology 221:731-739

48. Grazioli L, Morana G, Kirchin MA, Schneider G (2005) Accurate differentiation of focal nodular hyperplasia from hepatic adenoma at gadobenate dimeglumine-enhanced MR imaging: prospective study. Radiology 236:166-177

49. Grazioli L, Bondioni MP, Faccioli N et al (2010) Solid focal liver lesions: dynamic and late enhancement patterns with the dual phase contrast agent gadobenate dimeglumine. J Gastrointest Cancer 41:221-232

50. Morana G, Grazioli L, Kirchin MA et al (2011) Solid hypervascular liver lesions: accurate identification of true benign lesions on enhanced dynamic and hepatobiliary phase magnetic resonance imaging after gadobenate dimeglumine administration. Invest Radiol 46:225-239

51. Kim YK, Lee JM, Kim CS, Chung GH, Kim CY, Kim IH (2005) Detection of liver metastases: gadobenate dimeglumineenhanced three-dimensional dynamic phases and one-hour delayed phase MR imaging versus superparamagnetic iron oxide-enhanced MR imaging. Eur Radiol 15:220-228

52. Pediconi F, Catalano C, Occhiato R et al (2005) Breast lesion detection and characterization at contrast-enhanced MR mammography: gadobenate dimeglumine versus gadopentetate dimeglumine. Radiology 237:45-56

53. Pediconi F, Catalano C, Padula S et al (2008) Contrast-enhanced MR mammography: improved lesion detection and differentiation with gadobenate dimeglumine. AJR Am J Roentgenol 191:1339-1346

54. Martincich L, Faivre-Pierret M, Zechmann CM et al (2011) Multicenter, double-blind, randomized, intraindividual crossover comparison of gadobenate dimeglumine and gadopentetate dimeglumine for breast MR imaging (DETECT trial). Radiology 258:396-408

55. Gilbert FJ, van den Bosch HCM, Petrillo A et al (2014) Comparison of gadobenate dimeglumine-enhanced breast MRI and gadopentetate dimeglumine-enhanced breast MRI with mammography and ultrasound for the detection of breast cancer. $\mathbf{J}$ Magn Reson Imag 39:1272-1286

56. Jinzaki M, Tanimoto A, Narimatsu Y et al (1997) Angiomyolipoma: imaging findings in lesions with minimal fat. Radiology 205:497-502

57. Obuz F, Karabay N, Secil M et al (2000) Various radiological appearances of angiomyolipomas in the same kidney. Eur Radiol 10:897-899

58. Allen BC, Tirman P, Jennings Clingan M, Manny J, Del Gaizo AJ, Leyendecker JR (2014) Characterizing solid renal neoplasms with MRI in adults. Abdom Imag 39:358-387

59. Ng KL, Rajandram R, Morais C et al (2014) Differentiation of oncocytoma from chromophobe renal cell carcinoma (RCC): can novel molecular biomarkers help solve an old problem? J Clin Pathol 67:97-104

60. Langan RC, Ripley RT, Davis JL et al (2012) Liver directed therapy for renal cell carcinoma. J Cancer 3:184-190

61. Motzer RJ, Bander NH, Nanus DM (1996) Renal-cell carcinoma. N Engl J Med 335:865-875

62. Janzen NK, Kim HL, Figlin RA, Belldegrun AS (2003) Surveillance after radical or partial nephrectomy for localized renal cell carcinoma and management of recurrent disease. Urol Clin North Am 30:843-852

63. Staehler MD, Kruse J, Haseke N et al (2010) Liver resection for metastatic disease prolongs survival in renal cell carcinoma: 12 -year results from a retrospective comparative analysis. World J Urol 28:543-547

64. Belldegrun A, deKernion JB (1998) Renal tumors. In: Walsh PC, Retik AB, Vaughan ED Jr et al (eds) Campbell's urology, 7th edn. W.B.Saunders, Philadelphia, pp 2283-2326

65. Kim YK, Lee JM, Kim CS (2004) Gadobenate dimeglumineenhanced liver MR imaging: value of dynamic and delayed imaging for the characterization and detection of focal liver lesions. Eur Radiol 14:5-13

66. Kim YK, Lee JM, Kim CS, Chung GH, Kim CY, Kim IH (2005) Detection of liver metastases: gadobenate dimeglumineenhanced three-dimensional dynamic phases and one-hour delayed phase MR imaging versus superparamagnetic iron oxide-enhanced MR imaging. Eur Radiol 15:220-228

67. Fu GL, Du Y, Zee CS et al (2012) Gadobenate dimeglumineenhanced liver magnetic resonance imaging: value of hepatobiliary phase for the detection of focal liver lesions. J Comput Assist Tomogr 36:14-19

68. MultiHance summary of product characteristics. http://www. medicines.org.uk/emc/medicine/6132/SPC/multihance/. Accessed 20 April 2015

69. Kirchin MA, Lorusso V, Pirovano G (1048) Compensatory biliary and urinary excretion of gadobenate ion after administration of gadobenate dimeglumine (MultiHance $\left({ }^{\circledR}\right)$ ) in cases of impaired hepatic or renal function: a mechanism that may aid in the prevention of nephrogenic systemic fibrosis? Br J Radiol 2015(88):20140526

70. Paudyal B, Paudyal P, Tsushima Y et al (2010) The role of the $\mathrm{ADC}$ value in the characterisation of renal carcinoma by diffusion-weighted MRI. Br J Radiol 83:336-343

71. Razek AA, Farouk A, Mousa A, Nabil N (2011) Role of diffusion-weighted magnetic resonance imaging in characterization of renal tumors. J Comput Assist Tomogr 35:332-336

72. Yu X, Lin M, Ouyang H, Zhou C, Zhang H (2012) Application of ADC measurement in characterization of renal cell carcinomas with different pathological types and grades by $3.0 \mathrm{~T}$ diffusion-weighted MRI. Eur J Radiol 81:3061-3066

73. Sevcenco S, Heinz-Peer G, Ponhold L et al (2014) Utility and limitations of 3-Tesla diffusion-weighted magnetic resonance imaging for differentiation of renal tumors. Eur J Radiol 83:909-913

74. Sasamori H, Saiki M, Suyama J, Ohgiya Y, Hirose M, Gokan T (2014) Utility of apparent diffusion coefficients in the evaluation of solid renal tumors at 3T. Magn Reson Med Sci. 13:175-181

75. Wang H, Cheng L, Zhang $X$ et al (2010) Renal cell carcinoma: diffusion-weighted MR imaging for subtype differentiation at 3.0 T. Radiology 257:135-143

76. Beck SD, Patel MI, Snyder ME et al (2004) Effect of papillary and chromophobe cell type on disease-free survival after nephrectomy for renal cell carcinoma. Ann Surg Oncol 11:71-77 